\title{
Mal Nutrición por Exceso en Escolares
}

\author{
JACQUELINE IBARRA P. ${ }^{1}$, MARI ALARCÓN R. ${ }^{2}$ \\ 1. Nutricionista, Magíster en Salud Familiar. Departamento de Salud Pública. Facultad de Medicina. Universidad Católica \\ de la Santísima Concepción. \\ 2. Nutricionista, candidata a Magíster en Salud Pública. Departamento de Salud Pública. Facultad de Medicina. \\ Universidad Católica de la Santísima Concepción.
}

\begin{abstract}
Malnutrition by Excess Among School-Age Children

Objectives: To implement an educational intervention that contributes to the prevention of malnutrition by excess among adolescents. Patients and Method: This descriptive study included a sample of 939 individuals attending school at Colegio Manuel D'Alzon, in Lota (small town), between 2007-2009. Studies included anthropometric evaluations, nutritional intake and training workshops. Parents and teachers were included in the workshops. Healthy nutrition was incorporated in the curriculum, and a site for distribution of healthy foods was implemented. Results: A 3\% decrease in prevalence of malnutrition by excess was seen ( $28 \%$ to $25 \%$ ), most marked among males. This decrease was attributed to reduction in obesity and increase of lower weights. This decrease appears during a period of time when boys show a growth spurt, which occurs earlier among girls. Conclusion: Collaborative interventions between health and education show positive results in this population.
\end{abstract}

(Key words: Adolescent, nutritional status, obesity).

Rev Chil Pediatr 2010; 81 (6): 506-514

\section{RESUMEN}

Objetivo: Implementar una intervención educativa que contribuya a prevenir la malnutrición por exceso en adolescentes. Pacientes y Método: Estudio descriptivo observacional que incluyó una muestra de 939 personas pertenecientes a la comunidad educativa del Colegio Manuel D’Alzon de la Comuna de Lota, período 2007-2009. Se incluyo estudiantes a quienes se les realizó evaluaciones antropométricas, de ingesta alimentaria, y talleres educativos, se incluyó igualmente a docentes y apoderados con quienes se efectuaron talleres educativos. Se logró la incorporación de temas de alimentación saludable en las actividades curriculares, y la implementación de un kiosco saludable (tipo b). Resultados: Se observó una disminución en la prevalencia

Trabajo recibido el 19 de abril de 2010, devuelto para corregir el 25 de mayo de 2010, segunda versión el 02 de agosto de 2010, tercera versión el 08 de octubre de 2010, aceptado para publicación el 04 de noviembre de 2010.

Correspondencia a:

Jacqueline Ibarra P.

E-mail: jibarra@ucsc.cl 
de malnutrición por exceso en 3 puntos porcentuales de un $28 \%$ inicial a un $25 \%$ final, siendo en el género masculino en donde se hace más evidente esta reducción. Atribuyéndose esto al aumento del estado de bajo peso y reducción de la obesidad, ello se sustentaría en el peak que alcanza el estirón puberal en los varones en este período de vida, lo que en las niñas ocurre a lo menos dos a tres años antes. Conclusiones: Estas intervenciones colaborativas entre el sector salud y educación logran resultados positivos en disminuir la mal nutrición por exceso en la población escolar.

(Palabras clave: Adolescente, estado nutricional, obesidad).

Rev Chil Pediatr 2010; 81 (6): 506-514

\section{Introducción}

En Chile, la obesidad en los púberes es de un 33\%. ${ }^{1}$. El Ministerio de Salud (Minsal) realiza intervenciones que buscan reducir tal problemática en todo el ciclo vital, sin embargo, un grupo escasamente intervenido es el de los 13 a 18 años.

A fin de brindar intervenciones a esta población desde los años 90, la Organización Mundial de la Salud impulsa la iniciativa de Escuelas Promotoras de la Salud en América Latina, las que se encuentran enmarcadas dentro de la Promoción de la Salud.

El incorporar el componente educativo formal en el nuevo modelo de salud promocional, tiene como base el hecho de que el conjunto de comportamientos asociados a una vida sana, tiene mayor probabilidad de ser adoptado por los individuos y de perdurar a lo largo de sus vidas, cuando el aprendizaje comienza precozmente y existe un reforzamiento continuo.

De esta manera, los ambientes educacionales constituyen un lugar estratégico donde se pueden coordinar, integrar e implementar intervenciones de promoción de la salud y la búsqueda de mejoras de las condiciones de salud de la población escolar, profesores, educadores, administrativos, padres y miembros de la comunidad. Así mismo, permite mejorar las estrategias educativas y aumentar el potencial de aprendizaje de los alumnos.

Además de la familia, el entorno educacional es uno de los sistemas más poderosos para promover el conocimiento y la motivación necesaria para la adopción y mantención de estilos de vida saludables.
De ahí la importancia de incorporar acciones de promoción de la salud en el sistema educacional, desde que el niño ingresa al sistema hasta que termina su educación formal; integrando a dicho sistema al grupo familiar, en especial a los padres.

El Minsal en 1998 creó el "Consejo Nacional para la Promoción de la Salud -VIDACHILE", éste en conjunto con distintos sectores e instituciones diseñó un Plan estratégico de Promoción de Salud, en donde se establecieron metas, indicadores y estrategias para el período 2001-2010.

A pesar del desarrollo de dichas estrategias los problemas de mal nutrición por exceso no han disminuido, sino más bien van en aumento, lo que hace pensar que no sólo el componente "informacional" es importante, sino que está cobrando cada vez más relevancia la modificación de las prácticas, hábitos y costumbres alimentarias arraigadas fuertemente en nuestra población.

Identificada la necesidad de trabajar con este grupo etario y sumado a ello la responsabilidad social que posee la UCSC, se trabajó en el colegio Manuel D'Alzon de la comuna de Lota. Allí se efectuó un diagnóstico, nutricional e implementación de un proyecto de intervención en educación y comunicación social en Nutrición y Alimentación, de 2 años de duración, 2007-2009. El primer año se realizó el diagnóstico del estado nutricional de la población escolar, se realizó la aplicación de una encuesta alimentaria y se evaluó el nivel de conocimientos de estilos de vida saludable. Posteriormente, se realizó un ciclo de talleres a toda la comunidad educativa, estudiantes, do- 
centes, para-docentes y apoderados; abordando las temáticas de alimentación y nutrición tomando como base los contenidos del Manual de Alimentación y Nutrición para la enseñanza Básica elaborado por FAO, Ministerio de educación, Universidad de Chile. INTA año 2003.

El Objetivo de este estudio fue implementar una intervención educativa destinada a prevenir la malnutrición por exceso en adolescentes de un colegio de la Comuna de Lota, VIII Región.

\section{Sujetos y Métodos}

\section{Diseño}

Estudio descriptivo observacional que abarca el período comprendido entre los años 2007 a 2009 en toda la comunidad educativa pertenecientes al colegio Manuel D Alzon, establecimiento de enseñanza media de la comuna de Lota. El año 2007 se evaluaron e intervinieron los estudiantes que cursaban primero, segundo y tercero medio. Luego el año 2008 se evaluaron e intervinieron los estudiantes que cursaban cuarto año medio, y finalmente el año 2009 fueron evaluados e intervenidos los estudiantes que rendían tercero y cuarto año medio.

\section{Muestra}

Se incluyeron 939 personas constituidas de la siguiente manera:

- 472 estudiantes correspondiente al 90\% de los adolescentes matriculados, distribuidos en $1^{\circ}, 2^{\circ}, 3^{\circ}$ y $4^{\circ}$ año de enseñanza media, contemplándose una inasistencia de un $10 \%$ de los estudiantes a su actividad escolar normal.

- 419 padres y apoderados correspondientes al $80 \%$ de la totalidad de los cuatro niveles académicos del colegio.

- 48 docentes y directivos establecimientos correspondiente al $80 \%$ del total de la planta de recursos humanos del Colegio Manuel D`Alzon de la Comuna de Lota.

\section{Implementación:}

La ejecución del proyecto consideró distintas etapas:
Etapa 1: Difusión, motivación y sensibilización de toda la comunidad educativa.

Se efectuaron reuniones con los directivos, profesores, estudiantes del establecimiento a fin de generar la sensibilización, inquietud y posterior apoyo al correcto desarrollo de la investigación. En dichas reuniones se abordaron temáticas que incluían el actual estado de salud de la población chilena, resaltando el perfil epidemiológico centrado en enfermedades crónicas no transmisibles, generando la necesidad de incorporar al curriculum vigente temáticas de estilos de vida saludable.

\section{Etapa 2: Diagnóstico}

Se realizó el levantamiento de una línea de base para conocer en profundidad la realidad relacionada con la situación del estado nutricional e ingesta calórica como también el nivel de conocimientos en estilos de vida saludables existente en los estudiantes. Ello se realizó a través de mediciones antropométricas, aplicación de encuesta de tendencia de consumo modificada ${ }^{2}$ y la utilización de la encuesta "Vida Grossa" 3 del Minsal destinada a evaluar la percepción de estilos de vida en las condicionantes de alimentación saludable y actividad física. Los resultados permitieron organizar las temáticas que se desarrollaron con toda la comunidad educativa.

Las mediciones antropométricas de peso y talla se efectuaron en el año 2007 en todos los escolares ( $1^{\circ}, 2^{\circ}$ y $3^{\circ}$ medio), en el año 2008 se evaluó a aquellos estudiantes que cursaban $4^{\circ}$ medio y en el año 2009 se evaluó a los estudiantes que cursaban $3^{\circ}$ y $4^{\circ}$ medio respectivamente.

Las mediciones antropométricas fueron realizadas en condiciones estandarizadas, por estudiantes entrenados de tercer año de la carrera de nutrición de la UCSC. Los evaluados presentaban el mínimo de ropa, en las niñas y niños se estableció: polera de algodón manga corta, pantalón de buzo, ropa interior, calcetines y sin zapatillas. Se efectuó el posterior descuento por ropa considerando un peso de pantalón de $350 \mathrm{~g}$ y para la polera $185 \mathrm{~g}$.

Para conocer el peso y la talla se utilizó una balanza marca SECA con tallímetro incor- 
porado (precisión $100 \mathrm{~g} \mathrm{y} 0,1 \mathrm{~cm}$, respectivamente).

Con estas mediciones se evaluó el IMC/Edad y Talla/Edad de acuerdo a desarrollo puberal. Se clasificó a los niños como obesos si su IMC era mayor o igual a 95 de la referencia $\mathrm{CDC} /$ $\mathrm{NCHS}^{4}$.

En el caso de los directivos, docentes, paradocentes, administrativos y auxiliares a través de reuniones-talleres se evalúo el nivel de conocimientos acerca de temáticas de vida saludable.

Se realizó un análisis de resultados de las evaluaciones realizadas a estudiantes, como de la información reportada por los docentes, paradocentes y directivos.

\section{Etapa 3: Intervención}

Se trabajo con cada uno de los eslabones de la comunidad educativa con metodologías didácticas distintas.

Con los estudiantes de $1^{\circ}, 2^{\circ}, 3$ y $4^{\circ}$ se realizaron 4 talleres cuyos contenidos se basaron en el "Manual de alimentación y nutrición", elaborado por FAO Ministerio de Educación Universidad de Chile e INTA 2003. Siendo los contenidos temáticos abordados: alimentación saludable, necesidades nutricionales, nutrición y salud, alimentos sanos y seguros, y seguridad alimentaria familiar.

Esta actividad se desarrolló dentro de la jornada estudiantil normal por lo que la asistencia a ella fue de un $90 \%$.

En el transcurso del desarrollo de la investigación se efectuaron actividades de promoción: el montaje y desarrollo de una obra de teatro centrada en la alimentación y nutrición adecuada en la etapa de la adolescencia y la realización de frecuentes paneles murales alusivos a los estilos de vida saludable.

Dentro de la asignatura de educación física se logró la incorporación de la temática de alimentación saludable siendo desarrollada con metodologías teóricas y practicas.

El nivel de conocimientos acerca de temáticas de vida saludable de los docentes, directivos y paradocentes se evalúo a través de la realización de focus group.

Posterior a ello se realizaron reuniones ta1leres que abordaron la temática de alimentación saludable y kiosco saludable.
Para luego iniciar la motivación del cambio de kiosco tradicional a un kiosco saludable. Y lograr el apoyo para implementar un kiosco saludable tipo $\mathrm{B}^{5}$.

Con los padres y apoderados, se efectuaron actividades de sensibilización dejando de manifiesto la situación nutricional del colegio, para luego desarrollar actividades de promoción como la obra de teatro cuya temática se centraba en alimentación y nutrición adecuada en la etapa de la adolescencia. Sumado a ello se desarrollo taller donde se abordaron contenidos del "Manual de alimentación y nutrición", elaborado por FAO Ministerio de Educación Universidad de Chile e INTA 2003.

Para el análisis estadístico se calcularon los promedios y las desviaciones standard.

\section{Resultados}

En la figura 1 se muestra la población intervenida, que correspondió al 90\% de la matricula.

En dicha población en el año 2007 la prevalencia de malnutrición por exceso encontrada fue de un $28 \%$ y posterior a la intervención el año 2009 se obtiene una prevalencia de mal nutrición por exceso de $25 \%$ disminuyendo en 3 puntos porcentuales (figura 2). La mal nutrición por exceso considera la clasificación que incorpora los diagnósticos nutricionales de riesgo de obesidad y obesidad.

En la figura 3 se presenta la mal nutrición por déficit, la cual aumenta en 5 puntos porcentuales en el período evaluado. La mal nutrición por déficit se incrementó en las niñas en 2,66 puntos porcentuales. $Y$ en niños en 8,16 puntos porcentuales (figura 4).

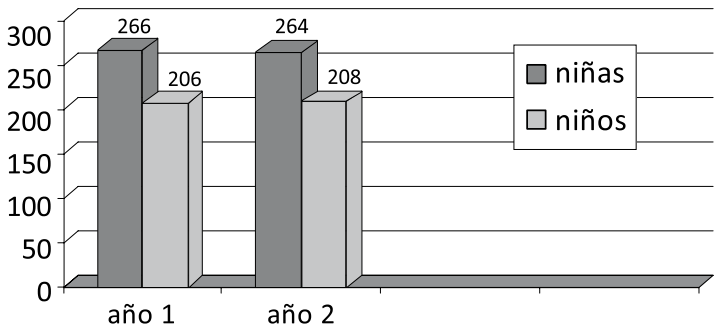

Figura 1. Población escolar total antes y post intervención según género del colegio Manuel D`Alzon de la comuna de Lota. 


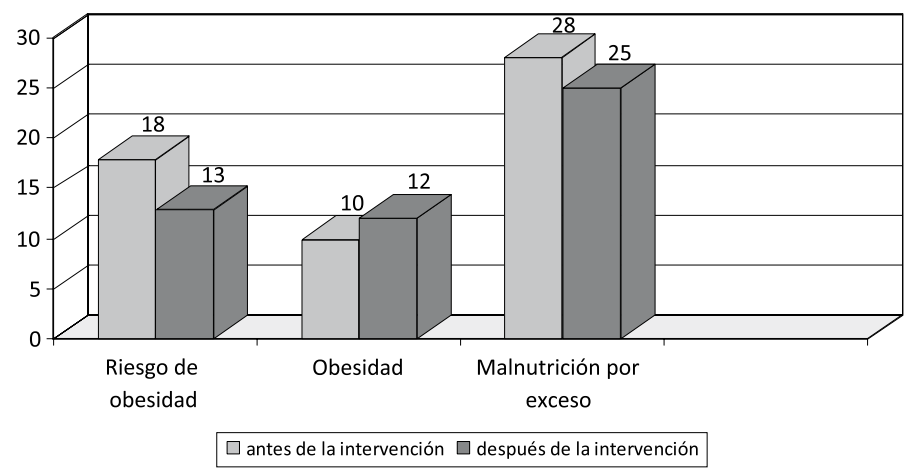

Figura 2. Malnutrición por exceso y sus componentes, estudiantes del colegio Manuel D`Alzon de la comuna de Lota.

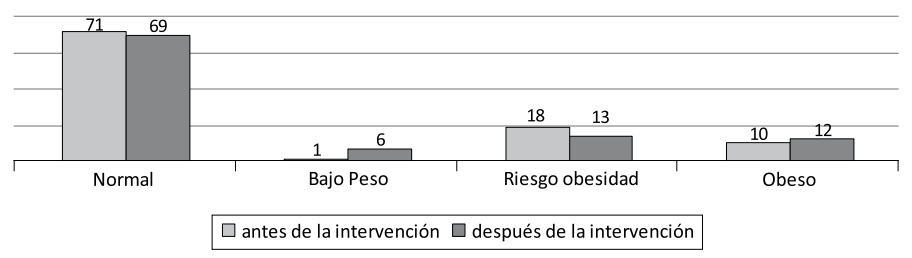

Figura 3. Estado nutricional de estudiantes del colegio Manuel D`Alzon de la comuna de Lota.

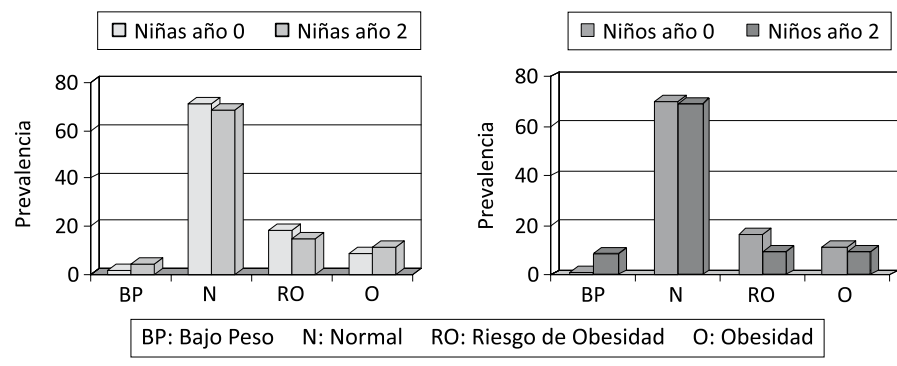

Figura 4. Estado nutricional según género de estudiantes del colegio Manuel D`Alzon de la comuna de Lota.

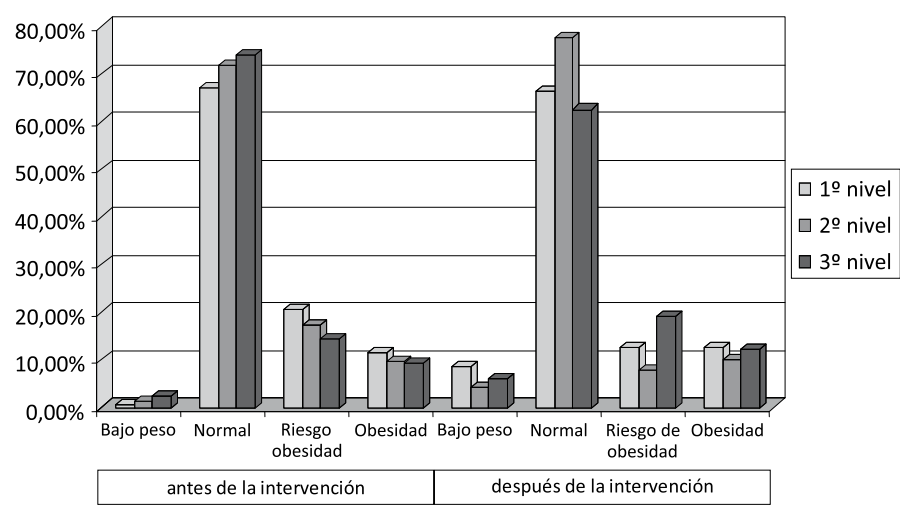

Figura 5. Estado nutricional según nivel de enseñanza media de estudiantes del colegio Manuel D`Alzon de la comuna de Lota.
En relación, al género la prevalencia inicial de mal nutrición por exceso de niñas fue de un $27,44 \%$ y de niños $28,64 \%$ y al finalizar el proyecto año 2009 la prevalencia de niñas es de $26,85 \%$ y niños $19,22 \%$. Por lo tanto, en niñas disminuyó en 0,59 puntos porcentuales y en niños 9,44 puntos porcentuales (tabla 1).

La prevalencia de malnutrición por exceso (figura 5) fue en el primer nivel (primeros medios) al inicio de la intervención de $32,1 \%$ y posterior a la intervención una prevalencia de $25,1 \%$, bajando en 7 puntos porcentuales. En el segundo nivel (segundos medios) fue de $26,9 \%$ para luego disminuir a un $18 \%$, es decir, se disminuye en 8,9 puntos porcentuales. En el tercer nivel (terceros medios) el año 2007 la prevalencia fue de $23,6 \%$, posterior a la intervención se observó una prevalencia de $31,2 \%$ lo que muestra un incremento porcentual de 7,6 puntos.

La prevalencia de malnutrición por déficit, (figura 5) se distribuye como sigue:

- Los primeros medios presentaron post intervención un incremento de 8 puntos porcentuales.

- Los segundos medios se observó un incremento en 3,1 puntos porcentuales.

- Los terceros medios se produce un incremento de 3,9 puntos porcentuales.

El requerimiento nutricional se calculó en base a actividad física ligera, considerando las clases de educación física de una vez a la semana impartidas por el colegio. Según valores de referencia de la Guía de Alimentación de 11 a 18 años del año 2006 elaborada por el INTA y la Universidad de Chile ${ }^{6}$. 
Tabla 1. Comparación del Estado Nutricional versus género en estudiantes. Colegio Manuel D`Alzon de Lota

\begin{tabular}{|c|c|c|c|c|c|c|c|c|}
\hline \multicolumn{9}{|c|}{$1^{\circ}$ medio } \\
\hline \multicolumn{5}{|c|}{ Antes de la intervención } & \multicolumn{4}{|c|}{ Después de la intervención } \\
\hline & $\begin{array}{l}\text { Bajo } \\
\text { Peso }\end{array}$ & Normal & $\begin{array}{l}\text { Riesgo } \\
\text { obesidad }\end{array}$ & Obesa & $\begin{array}{l}\text { Bajo } \\
\text { peso }\end{array}$ & Normal & $\begin{array}{c}\text { Riesgo } \\
\text { obesidad }\end{array}$ & Obesa \\
\hline Niñas (n) & 1 & 68 & 20 & 11 & 5 & 66 & 13 & 16 \\
\hline Niños (n) & 0 & 49 & 16 & 9 & 10 & 50 & 9 & 6 \\
\hline
\end{tabular}

\begin{tabular}{|c|c|c|c|c|c|c|c|c|}
\hline \multicolumn{9}{|c|}{$2^{\circ}$ medio } \\
\hline \multicolumn{5}{|c|}{ Antes de la intervención } & \multicolumn{4}{|c|}{ Después de la intervención } \\
\hline & $\begin{array}{l}\text { Bajo } \\
\text { Peso }\end{array}$ & Normal & $\begin{array}{l}\text { Riesgo } \\
\text { obesidad }\end{array}$ & Obesa & $\begin{array}{l}\text { Bajo } \\
\text { Peso }\end{array}$ & Normal & $\begin{array}{l}\text { Riesgo } \\
\text { obesidad }\end{array}$ & Obesa \\
\hline Niñas (n) & 1 & 68 & 16 & 9 & 1 & 71 & 10 & 9 \\
\hline Niños (n) & 1 & 52 & 13 & 7 & 6 & 58 & 3 & 8 \\
\hline \multicolumn{9}{|c|}{$3^{\circ}$ medio } \\
\hline \multicolumn{5}{|c|}{ Antes de la intervención } & \multicolumn{4}{|c|}{ Después de la intervención } \\
\hline & $\begin{array}{l}\text { Bajo } \\
\text { Peso }\end{array}$ & Normal & $\begin{array}{c}\text { Riesgo } \\
\text { obesidad }\end{array}$ & Obesa & $\begin{array}{l}\text { Bajo } \\
\text { peso }\end{array}$ & Normal & $\begin{array}{l}\text { Riesgo } \\
\text { obesidad }\end{array}$ & Obesa \\
\hline Niñas (n) & 2 & 53 & 13 & 4 & 5 & 45 & 17 & 6 \\
\hline Niños (n) & 1 & 44 & 6 & 8 & 3 & 37 & 8 & 10 \\
\hline
\end{tabular}

Tabla 2. Ingesta calórica versus requerimiento calórico de estudiantes. Colegio Manuel D Alzon de Lota

\begin{tabular}{|lcccccccc|}
\hline \multicolumn{4}{c}{ Antes de la intervención } & \multicolumn{4}{c|}{ Después de la intervención } \\
Género & $\begin{array}{c}\text { Ingesta } \\
\text { Promedio }\end{array}$ & $\begin{array}{c}\text { Desv. Est. } \\
\text { Ingesta }\end{array}$ & $\begin{array}{c}\text { Requeri- } \\
\text { miento }\end{array}$ & $\begin{array}{c}\text { Adecuación } \\
\text { Calórica }\end{array}$ & $\begin{array}{c}\text { Ingesta } \\
\text { Promedio }\end{array}$ & $\begin{array}{c}\text { Desv. Est. } \\
\text { Ingesta }\end{array}$ & $\begin{array}{c}\text { Requeri- } \\
\text { miento }\end{array}$ & $\begin{array}{c}\text { Adecuación } \\
\text { Calórica }\end{array}$ \\
Niñas & 2176,97 & 907,32 & 1950,00 & $111,63 \%$ & 2079,28 & 541,09 & 1950,00 & $106,63 \%$ \\
Niños & 2889,42 & 1185,05 & 2450,00 & $117,93 \%$ & 2378,85 & 860,90 & 2450,00 & $97,10 \%$ \\
\hline
\end{tabular}

La adecuación calórica se define como la relación entre al ingesta calórica y el requerimiento calórico. Y es considerada aceptable y normal dentro de un rango de valores mínimo y máximo, siendo de $90 \%$ a $110 \%$ respectivamente ${ }^{7}$.

Según tabla 2, la adecuación calórica en el grupo de las niñas fue pesquisada una disminución en 5 puntos porcentual, quedando dentro de los márgenes de normalidad de 90 al 110\%. Por otro lado, en los niños disminuyó a 20,83 puntos porcentual, quedando en la normalidad de 90 al $110 \%$.

Desde el punto de vista de los objetivos de este estudio que correspondían a evaluar el nivel de conocimiento de los estilos de vida saludables, ellos fueron evaluados a través de la aplicación de la encuesta "Tienes una vida Grossa", focalizado en las condicionantes de nutrición y actividad física. En el ítem de nutrición el 36\% de los estudiantes no establecía horarios de alimentación omitiendo alguna comida del día, por otro lado el $83 \%$ selecciona alimentos pocos o no saludables, $5 \%$ consume 4 ó más frutas al día y $52 \%$ refiere que la alimentación que recibe es con poca grasa (figura $6)$.

En el ítem actividad física, el $84 \%$ de los 


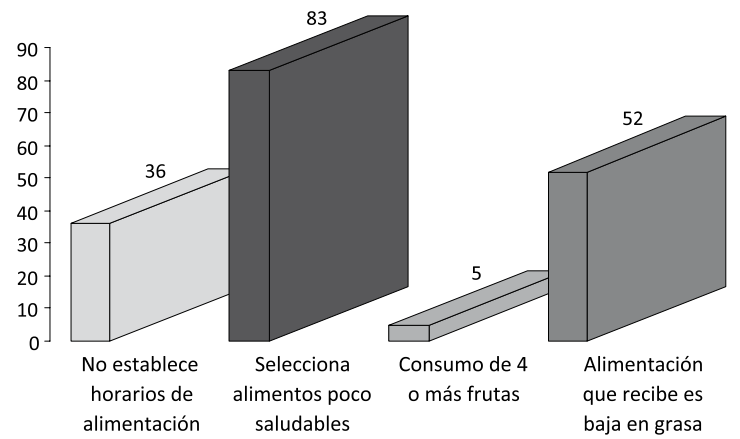

Figura 6. Encuesta "Tienes una vida grossa": Nutrición.

estudiantes permanece entre 1 a 3 horas mirando $\mathrm{TV}$, videos o en el computador, un $41 \%$ practica algún deporte y un $36 \%$ camina al menos más de 20 cuadras diarias (figura 7).

\section{Discusión}

Con el fin de favorecer el adecuado estado nutricional de los adolescentes, se incorpora dentro de las actividades curriculares habituales de la asignatura de educación física las temáticas de alimentación saludable, quedando pendiente y como una necesidad futura el aumento de horas destinadas a esta asignatura a objeto de disminuir el sedentarismo de la población objetivo. El acercamiento de los adolescentes a temas del área de alimentación y nutrición a través de los talleres educativos continuos y permanentes desarrollados durante el tiempo completo de la investigación permitió la constante entrega de información y a su vez el monitoreo del impacto a través de la evaluación antropométrica y dietaria que se realizó.

Los contenidos educativos que se abordaron en el desarrollo del estudio nos son ajenos a los estudiantes, dado que han sido entregadas a nivel curricular en la enseñanza básica, y se encuentran en las estrategias que continuamente el MINSAL entrega a toda la población.

Sin embargo, su utilización se sustentó en la brecha que existía entre el conocimiento adquirido por los estudiantes y las conductas alimentarias pesquisadas al inicio del estudio.

Tras el desarrollo de la investigación se lo-

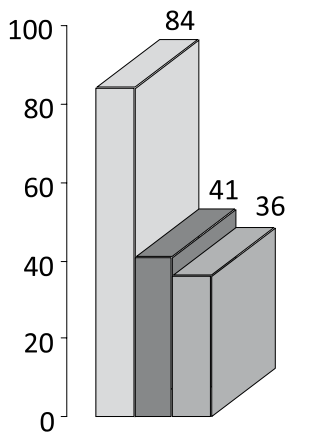

$\square 1$ a 3 horas en la TV

$\square$ Practica de algún deporte

$\square$ Camina más de 20 cuadras

Figura 7. Encuesta "Tienes una vida grossa": Actividad Física.

gró una internalización de dichos contenidos, lo que se refleja en la actual ingesta alimentaria que mantienen los adolescentes. En concordancia con las adecuaciones calóricas registradas al inicio del estudio $(114,7 \%)$ y luego de la intervención $(101,5 \%)$.

Actualmente la estrategia de "entornos educativos saludables" con su componente de kiosco saludable -educación- actividad física; no han sido evaluados en términos de impacto real frente a los indicadores de salud. Es posible afirmar que los resultados de encuestas y entrevistas aplicadas por el sector sanitario demuestran que el "nivel de conocimiento en temas de nutrición, salud y alimentación" ha mejorado, sin embargo, existe una clara discordancia entre "lo que el escolar sabe y lo que practica en su casa y escuela en términos de nutrición y alimentación", lo que evidencia un quiebre en lo que se está aprendiendo y el comportamiento adoptado. La gran tarea es hacer que estas temáticas de alimentación saludable, que se lograron incorporan en el currículo, faciliten el cambio entre el saber y hacer actual.

La implementación del kiosco saludable resultó una de las tareas mayor dificultad dentro del desarrollo de la investigación. El kiosco que originalmente presentaba el colegio se caracterizaba por el expendio de alimentos ricos en hidratos de carbono simples, grasas saturadas, sin ofrecer de alimentos del tipo saludable.

Ante la propuesta de cambio a kiosco tipo $\mathrm{C}$ existió resistencia por parte del sostenedor logrando sólo la implementación del kiosco tipo B. Esto por que los intereses de los sostenedores no necesariamente son concordantes 
con las estrategias de salud a nivel nacional. Dichos intereses incluyen temas netamente económicos y es esté el eje central de las dificultades que se producieron durante el proceso.

Ello genera la necesidad de implementar a través del Ministerio de Educación y Ministerio de Salud algún tipo de incentivo para aquellos establecimientos educacionales que instalen kioscos saludables tipo C. Todo ello dentro de la misión de asegurar un óptimo crecimiento y desarrollo de la población escolar.

Cualquier intervención que conjugue a la Salud Pública y a la Educación Chilena contribuye a mitigar el problema sanitario actual de epidemia de obesidad y resulta relevante. Al lograr mejorar los indicadores de salud en el escolar se genera un impacto a futuro, no sólo contribuyendo a mejorar la calidad de vida de la sociedad chilena sino que impactará absolutamente en el nivel de productividad y desarrollo de nuestro país.

El objetivo de este estudio fue diseñar, implementar a nivel local una metodología de intervención que contribuyese a prevenir y disminuir la mal nutrición por exceso en los niños y niñas en edad escolar (14 a 17 años).

Se levantó una línea de diagnóstico base de la situación nutricional-alimentaria de los adolescentes de 14 a 17 años de la comuna de Lota de la Octava Región de Chile, que se caracteriza por altos índices de desempleo y pobreza y que constituye una comuna socio-económicamente vulnerable y que habitualmente no es intervenida.

La prevalencia de mal nutrición por exceso en la muestra al inicio del estudio fue de $28 \%$ y tras la intervención esta disminuyó 3 puntos porcentuales quedando en $25 \%$.

Al realizar la revisión de la prevalencia de la mal nutrición por exceso en cada uno de los géneros se encuentra un descenso en el género masculino de 9,44 puntos porcentuales. En el caso de las adolescentes dicha baja es de $0,59 \%$ puntos porcentuales. Ello resulta coincidente con lo expuesto por Bellizi y colaboradores en el año 2001 en donde los varones adolescentes a medida que aumentaban en años disminuían la prevalencia de obesidad ${ }^{8}$.

En el estudio realizado en Santiago de Chile por Gaete y cols, se indicó la existencia hoy de un adelanto del desarrollo de las características sexuales secundarias en las niñas. Ello ocasiona que el estirón puberal ocurra antes en las niñas que en los varones (habitualmente en el período que finaliza la enseñanza básica). En el varón el estirón puberal ocurre un año a dos años después que en las niñas, generalmente en la enseñanza media afectando (en el caso de la población estudiada) el estado nutricional de normalidad que los varones tenían al inicio de la investigación y presentando al termino de ella mayor prevalencia de bajo de peso y una reducción de la malnutrición por exceso 9 . De este modo, en el segundo nivel de enseñanza media se observa dicha situación con el descenso de 10 puntos porcentuales del riesgo de obesidad de $17,3 \%$ a $7,8 \%$.

Consecuencia del estirón puberal el primer nivel presenta un alza del bajo peso, tras la intervención, de 8 puntos porcentuales (antes de la intervención era de $0,57 \%$ y después de la intervención es de 8,5\%).

En otra de las aristas de esta investigación, la coordinación con el VIDA CHILE a nivel de la comuna de Lota no se logró realizar dado que en este lugar el comité se encontraba temporalmente inactivo.

\section{Referencias}

1.- Ministerio de Salud de Chile: Norma Técnica de Evaluación Nutricional de Niños y Niñas de 6 a 18 años. 2004; 3.

2.- Rebolledo A: Encuestas Alimentarias. Rev Chil Nutr 1998; 25 (1): 28-34.

3.- Ministerio de Salud de Chile: Encuesta "Tienes un estilo de vida Grossa", Santiago, 2001. http://www. portaldeporivo.110mb.com/guias/VS.0006.pdf (consultado julio 2007).

4.- Barrera $G$ : Indicadores y Referentes para Evaluación del Estado Nutritivo, Crecimiento y Riesgo Metabólico. INTA 2006; 126-30.

5.- Ministerio de Salud de Chile: Documentos Técnicos. Kiosco Saludable, 2000. http://www.ego-chile.cl/Documentos/kiosco.pdf (consultado junio 2007).

6.- INTA: Guía de Alimentación de 11 a 18 años. http:// www.inta.cl/Consumidor/Nuevas/guia_adolescente.pdf (consultado junio 2007). 
IBARRA J. y cols.

7.- Thompson J, Manore M, Vaughan L: El papel de la nutrición en nuestra salud. En: "Nutrición”. Madrid, Ed. Pearson, 2008; 19-20.

8.- Bellizi M, Horgan G, Gillaume M, Dietz W: Prevalence of childhood and adolescent overweight and obesity in Asian and European countries. En Obesity in Childhood and Adolescenc, eds C Chen and W Dietz. Nestle Nutrition Workshops Series Pediatric Program, Vol 49. Philadelphia, Lippincott pag 23-35, 2001.

9.- Gaete X, Unanue N, Avila A, Cassorla F: Cambios en la edad de inicio de la pubertad en niñas de la comuna de Santiago: Implicancias para el diagnóstico de la pubertad precoz. Rev Chil Pediatr 2002; 73: 363-8.

10.- Fathalla M, Fathalla M: La redacción de un artículo científico. En "Guía práctica de investigación en salud", Washington DC. Publicación Científica y Técnica 2008; 620: 135-56.

11.- Taucher E: Bioestadística. Editorial Universitaria, p. 195-7, Santiago, 1997.

12.- Kovalasky I, Bay L, Rausch H, Berne E: Prevalencia de obesidad en una población de $10-19$ años en la consulta pediátrica. Rev Chil Pediatr 2005; 76: 324 -5. 